\title{
Perfil bibliométrico de la producción científica de la revista Odontología Sanmarquina, 2005-2014
}

\section{Bibliometric profile of scientific production in Odontología Sanmarquina journal, 2005-2014}

\begin{abstract}
Resumen
Objetivo: Evaluar un perfil bibliométrico de la producción científica de una revista odontológica peruana (Odontología Sanmarquina). Materiales y método: Estudio descriptivo que analizó los artículos científicos publicados en los volúmenes de la revista Odontología Sanmarquina en el intervalo de años 2005 hasta el 2014. Se analizó la productividad científica por año, el tipo, diseño y temática de los artículos así como la cantidad de autores, la filiación del primer autor, coautoría extranjera, el permiso bioético y el financiamiento de las investigaciones. La búsqueda de los artículos se realizó a través de un análisis manual vía el portal web de la revista. Resultados: Se analizaron 200 artículos publicados en un lapso de 10 ańos, el 59\% correspondió a artículos originales, el $25 \%$ a reportes clínicos y el 16\% a revisiones bibliográficas. Las temáticas más prevalentes de los estudios fueron las relacionadas a las ciencias básicas $(43,5 \%)$ y Periodoncia (13\%). Solo un 3,5\% de las publicaciones poseían coautoría extranjera y un $99,5 \%$ de los mismos carecían de un permiso bioético indicado dentro del artículo. Conclusiones: Los resultados indican que las ciencias básicas y la Periodoncia son la principal temática de publicación en la revista Odontología Sanmarquina y que la mayoría de las mismas son derivados de investigaciones originales.
\end{abstract}

Palabras clave: Bibliometría; Odontología; Perú.

\begin{abstract}
Objective: To evaluate a bibliometric profile of the scientific productivity in the journal Odontología Sanmarquina. Materials and method: A descriptive study was designed; the scientific articles published in the journal from the years 2005 to 2014 were analyzed. Scientific productivity per year, type, design and theme of the articles was analyzed and also the number of authors, the first author affiliation, foreign co-authorship, the bioethical permission and funding of these research. The search for articles was made through a manual analysis via the portal web of the journal. Results: 200 articles published over a period of 10 years were analyzed, 59\% were original articles, $25 \%$ clinical reports and literature reviews were the $16 \%$. The most prevalent themes of the studies were related to basic science $(43.5 \%)$ and Periodontology (13\%). Only 3.5\% of the publications had a foreign co-authored and $99.5 \%$ of them lacked a bioethical permission. Conclusions: The results indicate the basic sciences and periodontics is the main subject of publication in the journal, and that most of them are derived from original research. Keywords: Bibliometric, Dentistry, Peru.
\end{abstract}

Artículo Original

\section{Yuri Castro Rodríguez . .}

1 Facultad de Odontología. Universidad Nacional Mayor de San Marcos. Lima, Perú.

a Cirujano Dentista.

Correspondencia:

Yuri Alejandro Castro Rodríguez

Correo electrónico: yuricastro_16@hotmail.com Jr. Tomás Catari 463, Urb. El Trébol. Dpto. 201. Lima 39, Perú.

Conflicto de intereses: El autor declara no tener conflictos de interés.

Fuente de financiamiento: Ninguno.

Fecha de recepción: 14/04/17

Fecha de aceptación: 15/05/17

\section{Introducción}

La producción de conocimientos es punto clave para asegurar la eficacia y la calidad de la atención a la salud bucal y una formación y actualización de los profesionales que conduce al desarrollo del pensamiento crítico y forma parte de la cultura de una sociedad ${ }^{1}$.

Los conocimientos generados a partir de la investigación básica y su posterior evaluación a través de la investigación aplicada no deben quedarse como in- vestigaciones, deben seguir el proceso de la ciencia y llegar a convertirse en publicaciones científicas. La publicación científica permite reconocer el trabajo realizado por un investigador o grupo de investigadores; en este punto son las revistas científicas quienes cumplen el papel de ser los medios que permiten divulgar el conocimiento científico ${ }^{2}$. Toda revista científica debe cumplir ciertos protocolos: Deben utilizar el método de revisión por pares, tener por lo menos un ISSN y una indización internacional, ofrecer algún medio de visibilidad internacional, contar con un comité asesor, acreditar que su comité editor esté capacitado y mantener una periodicidad semestral como mínimo ${ }^{3}$.

En el Perú, existen múltiples revistas científicas con periodicidad de publicación en el área Odontológica, siendo las de mayor visibilidad según las bases de datos y directorios científicos las revistas: Odontología Sanmarquina, Estomatológica Herediana, Kiru, Visión 
Dental y Actualidad Odontológica y Salud. El análisis descriptivo de las publicaciones científicas de una revista se realiza a través de la bibliometría; los perfiles bibliométricos permiten: Medir el número de trabajos publicados sobre un tema, conocer la evaluación cronológica del ciertas disciplinas, conocer las revistas más relevantes de determinada disciplina, ubicar a los autores más productivos en las diferentes áreas del conocimiento, registrar la falta de información en ciertos temas, analizar la productividad científica de las instituciones y grupos de investigación y señalar tendencias, entre otros aspectos ${ }^{4-6}$. Permiten estar al tanto y valorar la producción científica de un área del conocimiento y entre sus principales aplicaciones se encuentra el área de la política científica, siendo además utilizados en los procesos de evaluación de los resultados de la actividad investigadora ${ }^{7}$. Estos estudios son escasos en el campo de la Odontología, sólo encontrándose antecedentes en Chile, Brasil, México, España y Colombia ${ }^{2,5,6}$.

En Perú no se registra un perfil de alguna revista de temática odontológica, no existiendo pues la posibilidad de observar desde un punto de vista crítico el desarrollo de la actividad científica; así como caracterizarlos desde el punto de vista cuantitativo y cualitativo ${ }^{8,9}$. En el presente estudio se tuvo como objetivo evaluar un perfil bibliométrico de la producción científica de la revista Odontología Sanmarquina en el periodo comprendido entre los ańos 2005-2014.

\section{Materiales y Método}

Se diseñó un estudio bibliométrico de la productividad científica de la revista Odontología Sanmarquina (Lima, Perú) en el intervalo de los años 2005-2014.

La búsqueda de la revista se realizó a través del portal del Sistema de Bibliotecas de la Universidad Nacional Mayor de San Marcos y a través de la página web de la misma revista.

Se realizó la selección y análisis de los artículos de cada volumen y número (la unidad de análisis fue el artículo científico); se incluyeron sólo publicaciones derivadas de investigaciones (artículos originales), artículos de revisión, series de casos y reportes de caso que se encontraron accesibles (ya sea en su versión HTML o PDF) en el intervalo del año 2005(enero) hasta el 2014 (diciembre) incluidos estos dos años. Se excluyeron publicaciones del tipo: Editoriales, cartas al editor, artículos en prensa, resúmenes de conferencias, re- señas y toda publicación que no posean introducción, materiales y método, resultados y discusión (IMRD). El análisis de los artículos se realizó a través de un observador (YC) con experiencia en realizar estudios bibliométricos; estabilidad temporal de 0,88.

Las variables analizadas incluyeron: Área de la revista (especializada o revista general), productividad (cantidad de publicaciones originales, de revisión y reportes de cada número en un determinado tiempo), filiación del primer autor, coautoría extranjera, financiamiento del estudio, aprobación de un comité de bioética, cantidad de citas bibliográficas, colaboración de estudiantes del pregrado, diseńo del artículo (de acuerdo a la metodología y categorizado como: Observacionales, experimentales, ensayos clínicos, y artículos de revisión) (Tabla 1) y temática de las publicaciones. Para la temática se consideró 10 clasificaciones: Ciencias básicas, Periodoncia, Implantología, Ortodoncia, Odontopediatría, Radiología, Cariología, Rehabilitación Oral, Endodoncia y Cirugía Bucomaxilofacial. Cada artículo se asignó a un solo tema. En los que pudiera existir un carácter mixto se seleccionó el factor temático predominante, para ello se accedió al contenido global del artículo si era necesario; en el resto se realizó con el título y el resumen. Los que no podían incluirse en ninguna categoría se consideraron inclasificables, quedando fuera del estudio temático.

Los datos fueron tabulados en el programa MS Excel 2003 (Microsoft Corporation, Redmond, USA) y analizados con el paquete estadístico SPSS 21. Se generó un análisis descriptivo con tablas de distribución para cada variable. El estudio no requirió de una aprobación por parte de un comité de ética ya que utilizó como material de estudio artículos publicados disponibles de manera pública.

\section{Resultados}

Se analizaron 10 volúmenes de la revista y un total de 200 publicaciones científicas que cumplieron los criterios de inclusión. Del total de artículos evaluados, 118 (59\%) correspondieron a publicaciones derivadas de investigaciones originales, $32(16 \%)$ de artículos de revisión y 50 (25\%) derivados de reportes de casos clínicos; encontrándose la mayor cantidad de publicaciones en el año 2008 con 23 artículos publicados (Tabla 2). El mayor porcentaje de publicaciones científicas correspondieron a estudios con un diseño observacional, 121 artículos (60,5\%), seguido de
Tabla 1. Apartados utilizados para la clasificación de la temática, tipos y diseńos de los artículos analizados

\begin{tabular}{cc}
\hline Temática de los artículos: & Tipos de artículos: \\
\hline 1. Ciencias básicas & Originales \\
$\begin{array}{c}\text { Anatomía, Histología, Patología, } \\
\text { Microbiología, Inmunología, } \\
\text { Educación para la salud, Ma- } \\
\text { teriales dentales, Historia de la } \\
\text { Odontologia, Odontología forense, } \\
\text { Farmacología. }\end{array}$ & Revisiones \\
2. Periodoncia & Deportes clínicos \\
3. Ortodoncia de estudio & Observacionales \\
Ortopedia & Experimentales \\
4. Odontopediatría & Ensayos clínicos \\
Pacientes con habilidades & Revisiones \\
especiales & \\
5. Cariología & \\
6. Rehabilitación Oral & \\
7. Radiología & \\
8. Cirugía Bucomaxilofacial & \\
9. Endodoncia & \\
10. Implantología & \\
\hline
\end{tabular}

Tabla 2. Productividad científica por año de la revista Odontología sanmarquina

\begin{tabular}{|c|c|c|c|c|c|c|c|}
\hline \multirow[t]{2}{*}{ Año } & \multicolumn{2}{|c|}{$\begin{array}{l}\text { Artículos } \\
\text { originales }\end{array}$} & \multicolumn{2}{|c|}{$\begin{array}{l}\text { Artículos de } \\
\text { revisión }\end{array}$} & \multicolumn{2}{|c|}{$\begin{array}{c}\text { Reportes } \\
\text { de caso }\end{array}$} & \multirow{2}{*}{$\begin{array}{l}\overrightarrow{0} \\
\underline{\underline{\Phi}}\end{array}$} \\
\hline & $\mathrm{n}$ & $\%$ & $n$ & $\%$ & $\mathrm{n}$ & $\%$ & \\
\hline 2005 & 8 & 40 & 8 & 40 & 4 & 20 & 20 \\
\hline 2006 & 10 & 53 & 4 & 21 & 5 & 26 & 19 \\
\hline 2007 & 14 & 74 & 3 & 16 & 2 & 10 & 19 \\
\hline 2008 & 14 & 61 & 2 & 9 & 7 & 30 & 23 \\
\hline 2009 & 11 & 50 & 2 & 9 & 9 & 41 & 22 \\
\hline 2010 & 10 & 53 & 3 & 15 & 6 & 32 & 19 \\
\hline 2011 & 10 & 53 & 2 & 10 & 7 & 37 & 19 \\
\hline 2012 & 14 & 70 & 3 & 15 & 3 & 15 & 20 \\
\hline 2013 & 13 & 68 & 1 & 6 & 5 & 26 & 19 \\
\hline 2014 & 14 & 70 & 4 & 20 & 2 & 10 & 20 \\
\hline Total & 118 & 59 & 32 & 16 & 50 & 25 & 200 \\
\hline
\end{tabular}

artículos derivados de investigaciones que manipularon a las variables independientes que fueron un total de 37 $(18,5 \%)$ estudios experimentales (Tabla 3). Con respecto a la temática de las publicaciones, la mayor cantidad de artículos publicados correspondió a ciencias básicas, 87 (43,5\%), seguida de artículos relacionados a Periodoncia, 26 (13\%) y Ortodoncia, 25 (12,5\%). La menor cantidad de publicaciones correspondieron a la temática de Radiología, 1 (0,5\%). Las publicaciones referidas a ciencias básicas abarcaron la mayoría de ensayos clínicos (seis artículos; uso de fármacos y agentes quimioterapéuticos) y estudios experimentales (21 artículos), seguido de los estudios relacionados a Cariología con cuatro y 
ocho artículos respectivamente (Tabla 3). La cantidad promedio de autores de los artículos evaluados fue de 3,94 $\pm 2,74$, con un mínimo de un autor y un máximo de 14 autores; la cantidad promedio de citas bibliográficas fue de $16,79 \pm 8$ (Tabla 4).

Con respecto a la filiación del primer autor de cada publicación, el 95,5\% de ellos correspondió a la nacionalidad peruana, siendo casi en su totalidad provenientes de la Universidad Nacional Mayor de San Marcos y uno proveniente de la Universidad San Luis Gonzales de Ica. El 3,5\% fueron provenientes de Brasil (Universidad Estadual de Campińas), y el 1\% provenientes de Argentina (Universidad del Nordeste de Buenos Aires). El 96,5\% de los artículos carecía de apoyo de un colaborador extranjero, siendo Brasil el principal país colaborador. El 99,5\% de las publicaciones no mencionaron un permiso bioético de aprobación de la investigación realizada. El $11 \%$ de los artículos evaluados sí mencionaron poseer financiamiento económico para la realización de las investigaciones; siendo la principal fuente de financiamiento la proveniente del Vicerrectorado de Investigación de la Universidad Nacional Mayor de San Marcos (Tabla 5). El 21,5\% de artículos poseían dentro de su autoría a un estudiante del pregrado.

\section{Discusión}

La medición del esfuerzo y repercusión de la actividad científica se basa en la bibliometría, su análisis es punto de partida para mejorar la productividad científica, ya que permite definir las líneas de investigación más pertinentes a partir de la correlación entre necesidades objetivas de investigación y número y calidad de artículos publicados ${ }^{10}$.

Conocer quiénes publican, dónde y acerca de cuáles temas permite describir el perfil bibliométrico de una institución, grupo de investigadores o de un país. Los 10 volúmenes evaluados de la revista Odontología Sanmarquina permiten describir datos de la realidad de la publicación científica derivada de la Universidad Nacional Mayor de San Marcos. La revista posee un periodo de publicación semestral con un rango de publicaciones entre 9-13 artículos por cada número, siendo la mayoría de estos artículos derivados de investigaciones originales y en menor medida los artículos derivados de revisiones bibliográficas. La producción anual de artículos de la revista osciló entre 19-23 artículos siendo un promedio de 20 artículos por año, tomando en consideración otras

Tabla 3. Distribución de la productividad científica por temática y diseńo de los artículos analizados

\begin{tabular}{|c|c|c|c|c|c|c|c|c|c|c|}
\hline \multirow{2}{*}{ Temática de los artículos } & \multicolumn{2}{|c|}{ Ensayos clínicos } & \multicolumn{2}{|c|}{ Experimentales } & \multicolumn{2}{|c|}{ Observacionales } & \multicolumn{2}{|c|}{ Revisiones } & \multicolumn{2}{|c|}{ Total } \\
\hline & $\mathrm{n}$ & $\%$ & $n$ & $\%$ & $\mathrm{n}$ & $\%$ & $\mathrm{n}$ & $\%$ & $\mathrm{n}$ & $\%$ \\
\hline Ciencias Básicas & 6 & 3 & 21 & 10,5 & 47 & 23,5 & 13 & 6,5 & 87 & 43,5 \\
\hline Periodoncia & 2 & 1 & 3 & 1,5 & 13 & 6,5 & 8 & 4 & 26 & 13 \\
\hline Ortodoncia & 0 & 0 & 2 & 1 & 22 & 11 & 1 & 0,5 & 25 & 12,5 \\
\hline Cariología & 4 & 2 & 8 & 4 & 5 & 2,5 & 5 & 2,5 & 22 & 11 \\
\hline Odontopediatría & 1 & 0,5 & 0 & 0 & 11 & 5,5 & 2 & 1 & 14 & 7 \\
\hline Rehabilitación Oral & 0 & 0 & 0 & 0 & 10 & 5 & 0 & 0 & 10 & 5 \\
\hline $\begin{array}{c}\text { Cirugía } \\
\text { Bucomaxilofacial }\end{array}$ & 0 & 0 & 0 & 0 & 7 & 3,5 & 0 & 0 & 7 & 3,5 \\
\hline Endodoncia & 0 & 0 & 3 & 1,5 & 1 & 0,5 & 0 & 0 & 4 & 2 \\
\hline Implantología & 0 & 0 & 0 & 0 & 4 & 2 & 0 & 0 & 4 & 2 \\
\hline Radiología & 0 & 0 & 0 & 0 & 1 & 0,5 & 0 & 0 & 1 & 0,5 \\
\hline Total & 13 & 6,5 & 37 & 18,5 & 121 & 60,5 & 29 & 14,5 & 200 & 100 \\
\hline
\end{tabular}

Tabla 4. Cantidad de autores y citas bibliográficas del total de artículos analizados

\begin{tabular}{|c|c|c|c|c|}
\hline & Media $\pm D E$ & Mínimo & Máximo & Rango \\
\hline \multicolumn{5}{|l|}{ Autores } \\
\hline & $3,94 \pm 2,74$ & 1 & 14 & 13 \\
\hline $\begin{array}{c}\text { Citas } \\
\text { bibliográficas }\end{array}$ & $16,79 \pm 8$ & 4 & 53 & 49 \\
\hline
\end{tabular}

revistas odontológicas latinoamericanas este promedio se encuentra por debajo de los perfiles bibliométricos encontrados en la Universidad de Concepción (Chile) ${ }^{11,12}$ y el perfil de la Universidad Autónoma de México ${ }^{5}$, sin embargo, esto puede deberse a que en otras revistas la periodicidad de publicación es bimensual y el algunos casos trimestral lo que aumentaría su productividad científica.

Los resultados señalan que el área que más publica son las relacionadas a las ciencias básicas (Fisiología, Farmacología, Microbiología, etc.), seguida de las publicaciones que se derivan de la temática periodontal. Esto concuerda con la tendencia actual que poseen las revistas científicas con mayor factor de impacto a nivel mundial. Las revistas "Dental Materials", "Journal Dental Research" y "Journal of Clinical Periodontology" actualmente gozan del mayor factor de impacto a nivel mundial, siendo su principal temática de publicación las ciencias básicas y Periodoncia ${ }^{13}$. Resalta la escasa producción científica de las áreas de Rehabilitación Oral, Cirugía, Endodoncia, Implantología y Radiología, esto señala una ausencia o falta de interés por investigar y publicar en dichas áreas dentro de la Facultad, no encontrándose en ninguno de ellos un artículo derivado de una investigación y solo limitándose a reportes clínicos o revisiones bibliográficas. "Una universidad que no investiga y no incentiva a la
Tabla 5. Filiación del primer autor, cooperación extranjera, permiso bioético y financiamiento económico de la totalidad de artículos analizados

\begin{tabular}{ccc}
\hline Variables & $\mathrm{n}$ & $\%$ \\
\hline Filiación del primer autor & & \\
Perú & 191 & 95,5 \\
Brasil & 7 & 3,5 \\
Argentina & 2 & 1 \\
Total & 200 & 100 \\
Coautoría extranjera & & \\
Sí & 7 & 3,5 \\
No & 193 & 96,5 \\
Total & 200 & 100 \\
Sí & & \\
No & 1 & 0,5 \\
Financiamiento & 199 & 99,5 \\
Sí & & \\
No & 22 & 11 \\
Total & 178 & 89 \\
& 200 & 100 \\
\end{tabular}

investigación será incapaz de analizar su realidad y su entorno, si no se publica es un esfuerzo que se desperdicia" ${ }^{14,15}$, lo que recuerda la necesidad de investigar en todas las áreas de la Odontología dentro del claustro universitario.

Los autores son las personas encargadas de redactar, corregir y enviar los artículos científicos a una revista científica, el caso de la revista Odontología Sanmarquina muestra un promedio de cuatro autores por artículos; esta realidad es encontrada comúnmente en otras revistas científicas, en las cuales el máximo encontrado es de 7 u $8^{16,17}$. Esto puede deberse a una falta en la "ética de autoría" al considerar a una mayor cantidad de autores de los que merecen ser; lo cual explicaría la gran cantidad de autores que se tiene en algunos artículos encontrados. Un punto interesante encontrado en el estudio es la filiación y coautoría extranjera de las publicaciones, la mayoría de artículos corresponden a autores peruanos y sin una 
colaboración extranjera; sin embargo, un porcentaje pequeño de los artículos derivaron de Brasil y Argentina. Esto es llamativo pues la revista actualmente carece de una indización a una base de datos lo que limita su visibilidad internacional y por ende una mayor colaboración de autores extranjeros que se animen a publicar en la revista. No se pudo analizar el factor de impacto de la revista al carecer de una indización a una base de datos, por lo que no fue posible evaluar la cantidad de citaciones internacionales de las publicaciones de la revista Odontología Sanmarquina, tampoco se logró realizar un parangón con otras revistas de circulación local y poder analizar los resultados con otras universidades.

\section{Conclusiones}

La productividad científica de la revista Odontología Sanmarquina se mantiene constante a lo largo de estos diez años de evaluación, siendo la mayor cantidad de publicaciones los artículos originales y los correspondientes a la temática de las ciencias básicas. Existe un elevado predominio de autores peruanos propios de la misma Universidad y Facultad de origen de la revista así como una aceptable participación de estudiantes de pregrado dentro de la autoría de los artículos publicados.

\section{Referencias bibliográficas}

1. Vilches A, Furió C. Ciencia, Tecnología, Sociedad: Implicaciones en la Educación Científica para el Siglo XXI". I Congreso Internacional "Didáctica de las Ciencias" y VI Taller Internacional sobre la Enseñanza de la Física. 6 al 10 de diciembre 1999 Centro de Convenciones Pedagógicas Cojimar, Ciudad de La Habana, Cuba. [consultado 03/04/2016]. Disponible en: http://www.oei.es/salactsi/ctseducacion.
2. Moraga J, Zuñiga A. Perfil bibliométrico ISI de la Facultad de Odontología de la Universidad de Concepción, 1989-2012. J Oral Res. 2013;2(1):18-22. DOI: $10.17126 /$ joralres.2013.004

3. Castro-Rodríguez Y. Perfil bibliométrico de la producción científica de una revista odontológica peruana: 2005-2014. Kiru. 2015;12(2):80-84.

4. Ramírez Godoy ME, Navarro E, Díaz Escoto A. Impacto de la producción editorial del Instituto Nacional de Psiquiatría Ramón de la Fuente, entre 1995 y 2006, de acuerdo con el ISI Web of Science. Salud Ment. 2008;31(1):3-17.

5. Lara N, López V, Mendoza S. La investigación odontológica en México. Rev Assoc Dent Mex. 2011;68(5):229-36.

6. Rodríguez BN, Llodra JC. Periodoncia en España, 2025. Estudio Dephi. Sociedad Española de Periodoncia 2011. Pp 93-102.

7. Abramo G, Ciriaco A, Di Costa F. National research assessment exercises: A comparison of peer review and bibliometrics rankings. Scientometrics. 2011;89(3):92941. DOI: $10.1007 / \mathrm{s} 11192-011$ 0459-x

8. Torres-Salinas D, López-Cozar ED, Jiménez-Contreras E. Ranking of departments and researchers within a university using two Different databases: Web of Science versus Scopus. Scientometrics. 2009;80(3):761-774. DOI:10.1007/s11192-008-21139.

9. Gutiérrez-Vela MM, Díaz-Haro A, Berbel-Salvador S, Lucero-Sánchez A, Robinson-García N, CutandoSoriano A. Bibliometric analysis of research on regenerative periodontal surgery during the last 30 years.
J Clin Exp Dent. 2012;4(2):e1128. DOI:10.4317/jced.50646.

10. García GA, García LA, Carreño T, Maldonado AE, Rojas L. La productividad científica de la Odontología en México. Rev Assoc Dent Mex. 2010;67(5):223-32

11. Cartes-Velásques R, Aravena TP. Perfil bibliométrico de la Odontología chilena, 2001-2010. Rev Clin Periodoncia Implantol Rehabil Oral. 2012;5(1):5-8. http:// dx.doi.org/10.4067/S071901072012000100001

12. Gálvez M. Publicaciones biomédicas: realidad de Chile y Latinoamérica. Rev Chil Radiol. 2006;12(3):113-7. http:// dx.DOI.org/10.4067/S071793082006000300003.

13. Alarcón M, Aquino C, Quintanilla C; Raymundo 1, Alvarez J. Odontología basada en evidencia: Las 82 revistas de mayor impacto. Int J Odontostomat. 2015;9(1):4352. http://dx.DOI.org/10.4067/ S0718-381X2015000100007.

14. Uribe SE, Henriquez N, Quinchalef P, Uribe D, Schuman W. Reporting quality of papers published in Chilean dental journals. Evaluation period: 2002-2012. J Oral Res. 2015;4(4):239-48. DOI. $10.17126 /$ joralres.2015.048

15. Jaime A, Rodríguez C, Aravena P. Methodological quality of clinical trials in pediatric dentistry research published in ISI journals, 20082012. J Oral Res. 2015;4(2):10915. DOI: $10.17126 /$ joralres. 2015.023

16. Moraga J. Level of evidence and geographic origin of articles published in Chilean dental journals. J Oral Res. 2014;3(1):36-9. DOI: $10.17126 /$ joralres.2014.010

17. Aravena P, Cartes-Velásquez R, Manterola C. Productividad y calidad metodológica de artículos clínicos en Cirugía Oral y Maxilofacial en Chile, 2001-2012. Rev Chil Cir. 2013;65(5):382-8. http:// dx.DOI.org/10.4067/S0718 